\title{
Valacyclovir im Anschluss an die Akuttherapie kann das Outcome nicht verbessern
}

Fragestellung: Die Studie sollte klären, ob bei Patienten mit einer Herpes-simplex-Enzephalitis die Gabe von Valacyclovir über 90 Tage im Anschluss an eine übliche Akuttherapie mit Aciclovir zu einem verbesserten Langzeit-Outcome führt.

Hintergrund: Die Mortalität der Herpes-simplex-Enzephalitis konnte durch die wirksame Akuttherapie mit Aciclovir auf allerdings immer noch $14-19 \%$ signifikant gesenkt werden. Unter den Überlebenden gibt es jedoch sehr viele Patienten, die neurologische und vor allem auch neuropsychologische Defizite nach überstandener Akutphase aufweisen und dadurch in ihrem alltäglichen Leben oder auch an der Rückkehr ins Berufsleben gehindert werden. Bisherige zusätzliche Therapien beschränkten sich vornehmlich auf rehabilitative Maßnahmen. Inwieweit die Gabe von Kortikosteroiden zu einer Besserung führen könnte, ist ebenfalls noch unklar.

Gnann JW, Sköldenberg B, Hart J, et al; National Institute of Allergy and Infectious Diseases Collaborative Antiviral Study Group. Herpes simplex encephalitis: lack of clinical benefit of lon-term valacyclovir therapy. Clin Infect Dis 2015: 61: $683-91$
Patienten und Methodik: Insgesamt wurden 87 Patienten mit einer im Liquor mittels HSV-PCR nachgewiesenen Herpes-simplex-Enzephalitis in diese doppelblind, randomisierte, amerikanische Multicenterstudie eingeschlossen. Es wurde entwe- der Valacyclovir ( 3 x 2 g/Tag) $(\mathrm{n}=40)$ oder Placebo $(\mathrm{n}=47)$ als Tabletten für 90 Tage oral verabreicht. Primärer Endpunkt war das Überleben mit keinen oder nur geringen neuropsychologischen Defiziten gemessen an der Mattis Dementia Rating Scale (MDRS) nach zwölf Monaten.

Ergebnisse: Die eingeschlossenen Patienten waren im Median 55 Jahre alt, $54 \%$ männlichen Geschlechts und 95\% kaukasischer Abstammung. $64 \%$ der Patienten hatten vor Beginn der Studie keine oder nur milde neuropsychologische Defizite im MDRS. Am Ende der Gabe der Studienmedikation nach 90 Tagen erreichten $84 \%$ den primären Endpunkt, nach zwölf Monaten wiesen $88 \%$ der Patienten keine oder nur geringe neuropsychologische Defizite auf, nach 24 Monaten 89,5\%. Es gab dabei zu keinem der Follow-up-Untersuchungen signifikante Unterschiede zwischen Verum und Placebo. Der Mini-Mental-StateTest lag nach 90 Tagen bei $88 \%$ zwischen 23 und 30 Punkten und nach 24 Monaten bei $91 \%$.

Schlussfolgerungen: Die zusätzliche Gabe von Valacyclovir bis 90 Tage nach Ende der Akuttherapie führte in dieser Studie zu keinem zusätzlichen Benefit bezogen auf die messbaren neuropsychologischen Defizite. Insgesamt wurde dabei die Medikation gut vertragen, schwerwiegende Nebenwirkungen traten nicht auf. Lediglich gastrointestinale Nebenwirkungen waren in der Verumgruppe häufiger.

\section{- Kommentar von Matthias Maschke, Trier}

\section{Einfluss antiviraler Substanzen auf das Outcome weiter untersuchen}

Die Studienergebnisse waren für die ausgesuchte Patientengruppe komplett negativ, sodass die zusätzliche Gabe von Valacyclovir nach Ende der Akuttherapie mit Aciclovir nicht empfohlen werden kann. Zu beachten ist allerdings, dass die Patienten mit den schwersten Herpes-simplex-Enzephalitiden nicht eingeschlossen waren, da sie entweder schon in der Akutphase verstorben waren oder die Medikation nicht schlucken konnten. Insofern hatten die eingeschlossenen Patienten an sich bereits eine bessere Prognose bezüglich der Rückbildung neuropsychologischer Defizite. Darüber hinaus könnte man am primären Endpunkt keine oder milde neuropsychologische Defizite kritisieren, dass bereits milde neuropsychologische Defizite die Ausübung des vormaligen Berufs verhindern könnten.

Neuere Studie weisen darauf hin, dass nach überstandener Akutphase persistierende inflammatorische, vor allem autoimmun getriggerte Prozesse die Rekonvaleszenz behindern oder sogar zu einer Verschlechterung führen können [1]. Unter anderem scheint eine Untergruppe von Patienten An- tikörper gegen NMDA-Rezeptoren zu entwickeln [1, 2]. Daher sind weitere Studien, die den Einfluss antiviraler oder auch immunsuppressiver Substanzen nach Abschluss der Akuttherapie auf das Outcome untersuchen, sinnvoll.
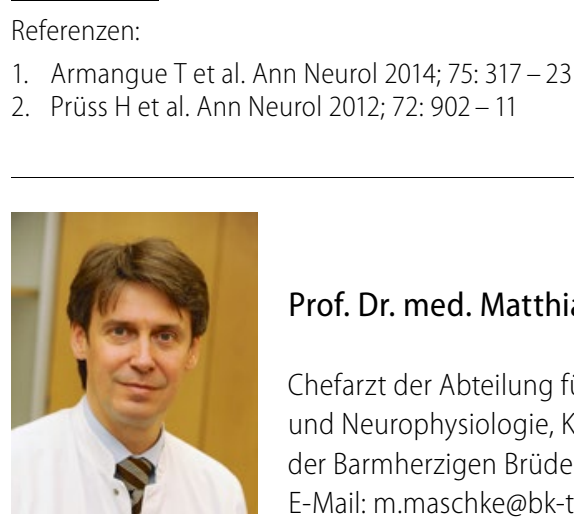

Prof. Dr. med. Matthias Maschke, Trier

Chefarzt der Abteilung für Neurologie und Neurophysiologie, Krankenhaus der Barmherzigen Brüder Trier E-Mail: m.maschke@bk-trier.de 